\title{
Functional and Pathological Analysis of Biological Systems Using Vibrational Spectroscopy with Chemometric and Heuristic Approaches
}

\author{
Aidan Meade \\ Technological University Dublin, aidan.meade@tudublin.ie \\ Colin Clarke \\ Dublin City University \\ Franck Bonnier \\ Technological University Dublin, Franck.Bonnier@tudublin.ie
}

See next page for additional authors

Follow this and additional works at: https://arrow.tudublin.ie/scschphyart

Part of the Physics Commons

\section{Recommended Citation}

Meade, A.D. et al. (2009) Functional and Pathological Analysis of Biological Systems Using Vibrational Spectroscopy with Chemometric and Heuristic Approaches. First workshop on Hyperspectral Image and Signal Processing: Evolution in Remote Sensing, pp.111-114. doi:10.1109/WHISPERS.2009.5288989

This Article is brought to you for free and open access by the School of Physics \& Clinical \& Optometric Science at ARROW@TU Dublin. It has been accepted for inclusion in Articles by an authorized administrator of ARROW@TU Dublin. For more information, please contact arrow.admin@tudublin.ie, aisling.coyne@tudublin.ie, gerard.connolly@tudublin.ie.

Funder: HEA Technology Sector Research Strand 3, HEA PRTLI Cycle 4 (National Biophotonics and Imaging Platform of Ireland)

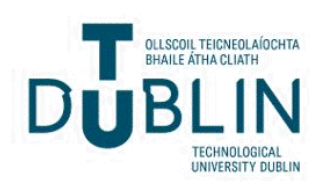




\section{Authors}

Aidan Meade, Colin Clarke, Franck Bonnier, Kelvin W. Poon, Amaya Garcia, Peter Knief, Kamila Ostrowska, Lorenzo Salford, Haq Nawaz, Fiona Lyng, and Hugh Byrne 


\title{
FUNCTIONAL AND PATHOLOGICAL ANALYSIS OF BIOLOGICAL SYSTEMS USING VIBRATIONAL SPECTROSCOPY WITH CHEMOMETRIC AND HEURISTIC APPROACHES
}

\author{
Meade A.D. ${ }^{1,3}$, Clarke, C. ${ }^{4}$, Bonnier, F. ${ }^{2,3}$, Poon, K. ${ }^{2,3}$, Garcia, A. ${ }^{2}$, Knief, P. ${ }^{2}$, Ostrowska, K. ${ }^{2}$, Salford, \\ L. $^{2}$, Nawaz, H. ${ }^{2}$, Lyng F.M. ${ }^{2,3}$ Byrne, H.J. ${ }^{2}$ \\ 1. School of Physics, Dublin Institute of Technology, Dublin, Ireland; \\ 2. FOCAS Research Institute, Dublin Institute of Technology, Dublin, Ireland; \\ 3. Radiation and Environmental Science Centre (RESC), FOCAS Research Institute, Dublin Institute of \\ Technology, Dublin, Ireland; \\ 4. National Institute for Cellular Biotechnology, Dublin City University, Dublin, Ireland
}

\begin{abstract}
Vibrational spectroscopy (Raman and FTIR microspectroscopy) is an attractive modality for the analysis of biological samples since it provides a complete noninvasive acquisition of the biochemical fingerprint of the sample. Studies in our laboratory have applied vibrational spectroscopy to the analysis of biological function in response to external agents (chemotherapeutic drugs, ionising radiation, nanoparticles), together with studies of the pathology of tissue (skin and cervix) in health and disease. Genetic algorithms have been used to optimize spectral treatments in tandem with the analysis of the data (using generalized regression neural networks (GRNN), artificial neural networks (ANN), partial least squares modelling (PLS), and support vector machines (SVM)), to optimize the complete analytical scheme and maximize the predictive capacity of the spectroscopic data. In addition we utilise variable selection techniques to focus on spectral features that provide maximal classification or regression of the spectroscopic data against analytical targets. This approach has yielded interesting insights into the variation of biochemical features of the biological system with its state, and has also provided the means to develop further the analytical and predictive capabilities of vibrational spectroscopy in biological analysis.
\end{abstract}

Index Terms - Vibrational spectroscopy, nanocytotoxicity, chemotherapeutic drugs, radiobiology, chemometrics, heuristic techniques.

\section{INTRODUCTION}

The drive in modern biological science for measurements of the complete state of a system has led to the development of high-content, high-throughput systems, with concurrent developments in analytical techniques [1].
Vibrational spectroscopy has been exploited for its ability to provide measurements of the complete biochemical signature of biological systems at high spatial resolution[2], giving the potential to generate pseudo-images denoting biochemical composition of the sample[3].

Studies at the Focas Research Institute have applied vibrational spectroscopy both to the analysis of tissue and cell pathology, and to the analysis of function in the cell. Early studies in the Institute involved the analysis of fixation effects in tissues with vibrational spectroscopy [4, 5], and the application of the technique to the analysis of pathology in cervical tissue [6]. The latter study has been extended to the discrimination of cellular samples within cervical smears (see results).

Recent advances in nanotechnology have seen functionalized nanoparticles used as agents in diagnostic imaging[7, 8]. Advances in the field of nanotoxicology, however, have recently identified potential risks and hazards associated with exposure to nanoparticles[9]. We have used vibrational spectroscopy to understand the mechanism of the interaction of quantum nanoparticles (quantum dots (QD's) and single walled carbon nanotubes SWCNT's) with biological systems, using other imaging techniques (confocal microscopy, fluorescence microscopy) as references [10]. This approach has also been used to develop vibrational spectroscopy as a methodology for the assessment of chemotherapeutic drug efficacy, while separate studies have been applied to the development of the technique and associated analytical methodologies to the analysis of radiobiological effect in-vitro. The present paper summarises aspects of our approach to the development of vibrational spectroscopy as an analytical platform, with specific reference to chemometric and heuristic techniques employed in the work. 


\section{METHODS}

\subsection{Raman Micro-spectroscopy}

For nanotoxicological studies of QD's, Raman spectra were acquired on a Horiba Jobin-Yvon HR800 (Villeneuve d'Ascq, France) LabRam instrument equipped with an excitation laser operating at $785 \mathrm{~nm}$ with a grating of 300 lines $/ \mathrm{mm}$. For studies on SWCNT spectra were acquired using a LabRam 1B instrument with an excitation at $514 \mathrm{~nm}$ with a grating of 1800 lines $/ \mathrm{mm}$. For studies of cellular pathology, spectra were recorded with a LabRam HR600 instrument operating at $532 \mathrm{~nm}$ using a grating of 600 lines $/ \mathrm{mm}$.

\subsection{FTIR Micro-spectroscopy (FTIRM)}

For studies on keratinocyte radiobiology FTIRM was performed using a Perkin-Elmer GX-II spectrometer. Spectra were recorded over a wavenumber range from 720 $\mathrm{cm}^{-1}$ to $4000 \mathrm{~cm}^{-1}$ with an aperture size of $100 \mu \mathrm{m} \times 100 \mu \mathrm{m}$, at a spectral resolution of $4 \mathrm{~cm}^{-1}$ and with 64 scans per spectrum. All spectra were recorded in transreflection mode; approximately 300 spectra were recorded at each dose and time point.

\subsection{Confocal Microscopy}

Confocal fluorescence microscopy was performed using a Zeiss LSM 510 META Confocal Laser Scanninng Microscope. The system offers 6 excitation wavelengths $(458 \mathrm{~nm}, 477 \mathrm{~nm}, 488 \mathrm{~nm}, 514 \mathrm{~nm}, 543 \mathrm{~nm}$ and $633 \mathrm{~nm})$ and for detection, three separate reflected light PMTs, each with its own adjustable pinhole and emission filter wheel. Lysotracker (511 nm fluorescence peak emission) probes were purchased from Invitrogen, (Carlsbad, CA, USA), and used to localize the cellular lysosomes. The Lysotracker probes are fluorescent acidotropic probes for labeling acidic organelles in live cells.

\subsection{Chemometric and Heuristic Techniques}

Various chemometric approaches have been adopted here, which largely depend on whether the objective of the analysis is to understand the spectroscopic changes occurring with exposure to external agents (PLS, GRNN and SVM regression) or classification of spectral content (PCA). Genetic algorithms have also been utilized to optimize spectral pre-processing, and in some cases for selection of variables of interest.

\section{RESULTS}

\subsection{Radiobiological Analysis}

FTIR spectra were regressed against radiation dose using linear PLS regression (PLSR), non-linear PLS (NL-PLSR incorporating a $2^{\text {nd }}$ order polynomial) and GRNN algorithms in order to evaluate the performance of the models in predicting radiation dose, and to identify features of interest in the spectra with respect to radiobiological damage. Table 1 summarizes the performance of the algorithms. Spectral features of radiobiological interest have been isolated using PLS Jack-knifing (results not shown). The prediction performance has also been increased with the use of SVM's and selection of optimal preprocessing with a genetic algorithm (unpublished data). Genetic algorithms have also been used for variable selection, and have improved the algorithm performance and interpretability.

\begin{tabular}{cllllll}
\hline $\begin{array}{c}\text { Time } \\
(\mathrm{hrs})\end{array}$ & $\begin{array}{l}\text { GRNN } \\
(\text { Cal. })\end{array}$ & $\begin{array}{l}\text { GRNN } \\
(\text { Pred. })\end{array}$ & $\begin{array}{l}\text { PLSR } \\
(\text { Cal. })\end{array}$ & $\begin{array}{l}\text { PLSR } \\
\text { (Pred.) }\end{array}$ & $\begin{array}{l}\text { NLPLSR } \\
(\text { Cal. })\end{array}$ & $\begin{array}{l}\text { NLPLSR } \\
\text { (Pred.) }\end{array}$ \\
6 & 0.025 & 0.037 & 0.19 & 0.28 & 0.35 & 0.53 \\
& $(0.009)$ & $(0.008)$ & $(0.01)$ & $(0.02)$ & $(0.03)$ & $(0.04)$ \\
12 & 0.291 & 0.462 & 0.66 & 0.82 & 0.64 & 0.81 \\
& $(0.021)$ & $(0.071)$ & $(0.03)$ & $(0.04)$ & $(0.03)$ & $(0.06)$ \\
24 & 0.094 & 0.185 & 0.22 & 0.33 & 0.28 & 0.45 \\
& $(0.012)$ & $(0.039)$ & $(0.01)$ & $(0.02)$ & $(0.01)$ & $(0.05)$ \\
48 & 0.087 & 0.234 & 0.32 & 0.48 & 0.31 & 0.46 \\
& $(0.005)$ & $(0.057)$ & $(0.02)$ & $(0.02)$ & $(0.01)$ & $(0.02)$ \\
96 & 0.003 & 0.007 & 0.29 & 0.37 & 0.40 & 0.55 \\
& $(0.0002)$ & $(0.002)$ & $(0.02)$ & $(0.03)$ & $(0.03)$ & $(0.03)$ \\
\hline
\end{tabular}

Table 1. Calibration and prediction performances of GRNN, PLSR and NL-PLSR models in prediction of dose with time point postirradiation (Calibration and prediction RMSE's are in the units of radiation dose (Gray, Gy)). Figures in brackets denote the standard deviations on the mean.

\subsection{Nanotoxicology}

The nanoscrystal QDs (“625QD's” with $625 \mathrm{~nm}$ fluorescence peak emission) used in this study were purchased from Evident Technology, (Troy, N.Y., USA). The QDs have a core of CdSe, a shell of $\mathrm{ZnS}$ and a coating of polyethylene-glycol (PEG). Fluorescence and confocal microscopy shows that there are internalization of the QDs in $\mathrm{HaCaT}$ keratinocytes [11] after 1 hour exposure and the QDs are possibly retained in lysosomes. This is confirmed by colocalization of green LysoTracker probes and 625QD's (Fig. 1). Raman hyperspectral maps, generated from the anti-Stokes scatter signal from a hybrid confocal two-photon fluorescence microscopy scheme, were recorded at an excitation wavelength of $785 \mathrm{~nm}$. 

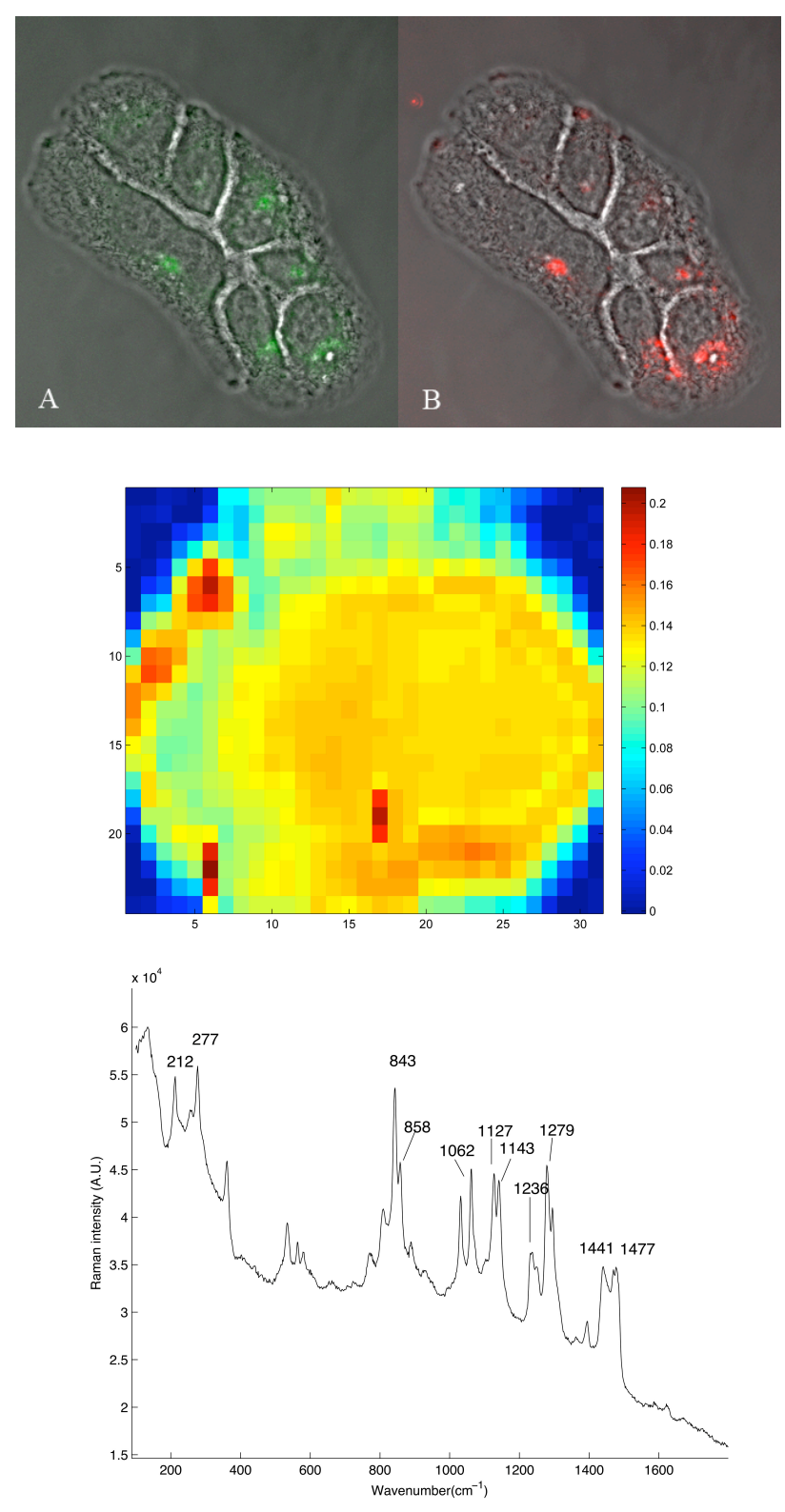

Fig. 1. Top panel: Confocal images of HaCaT cell with (left) green Lysotracker (A) and with (right) 625QDs (B) demonstrating the localization of the particle within the lysosomes;

Bottom panel: Top: Hyperspectral image (generated from fluorescence signal in anti-Stokes scatter spectra with $785 \mathrm{~nm}$ excitation) demonstrating localization of QD's (red hotspots) within an individual $\mathrm{HaCaT}$ cell using two-photon fluorescence spectroscopy; Bottom: QD spectrum extracted from hotspot positions;

Raman spectroscopy (at $514.5 \mathrm{~nm}$ excitation) has also been demonstrated to provide the means to localize SWCNTs within A549 human epithelial adenocarcinoma, as shown in figure 2. Hyperspectral images (see figure 2) generated from the G-line vibration in SWCNT's provides the means to non-invasively localize the nanoparticle within the cell, which is not possible from the visible images. This also potentially provides the means to non-invasively study the chemical interaction between the particle and the biological system at a high spatial resolution.
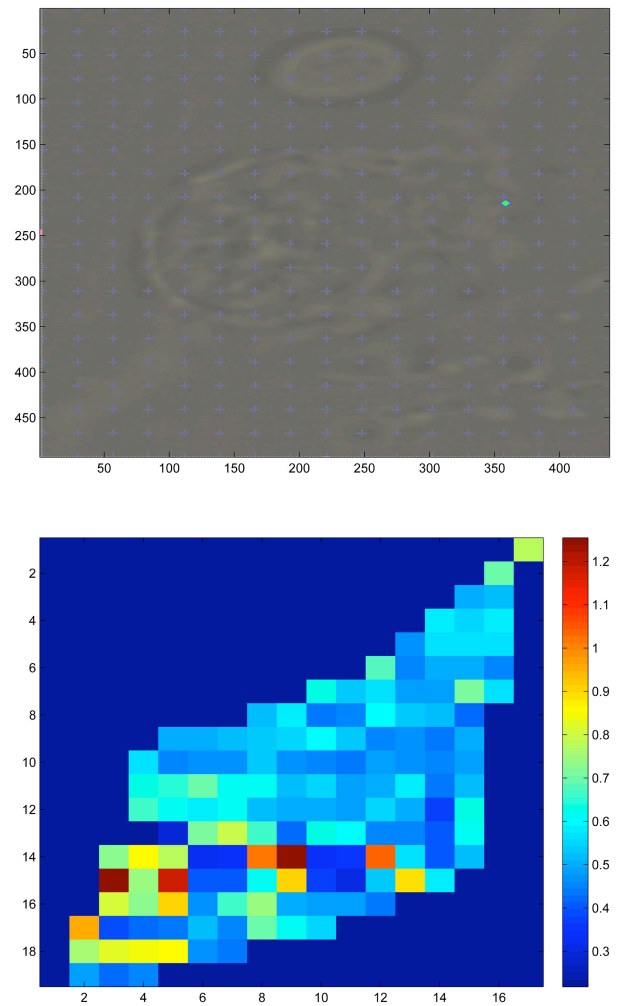

Fig. 2. Top, phase contrast of A549 cell exposed to SWCNT's; Bottom, hyperspectral image generated from G-line intensity $\left(\sim 1589 \mathrm{~cm}^{-1}\right)$. Scale in top image is by pixel number and bottom image is microns.

\subsection{Non-Invasive Tissue Histopathology}

Raman spectra recorded from cervical smear constituents contain features associated with each of the major components of the sample. PCA was applied to discriminate the signals recorded from the cytoplasm of epithelial cells, cytoplasm of navicular cells and vacuoles in the cytoplasmic area of navicular cells. The results are shown in figure 4, and show very good separation of the groups of Raman spectra. Signals from the cytoplasm of epithelial cells and cytoplasm of navicular cells, which are difficult to distinguish from a visual spectral analysis, are separated in the principal component scatter plot. Points representing spectra of the vacuoles filled with glycogen found in navicular cells exhibit incoherence in the PCA plot (Fig. 3), mainly due to various amount of glycogen in the vacuoles. 
This is an example of how the PCA technique adds discriminatory potential where analysis based on comparison of assigned peaks fails. This procedure may be used, for instance, when a polymorph overlaps with an epithelial cell, resembling a nucleus of an epithelial cell, as under the microscope these two structures are indistinguishable when physically overlapping. In this case, the signal from a polymorph can be classified according to the fingerprint spectrum, and subsequently excluded from a group of epithelial cell nuclei taken into account for diagnosis.

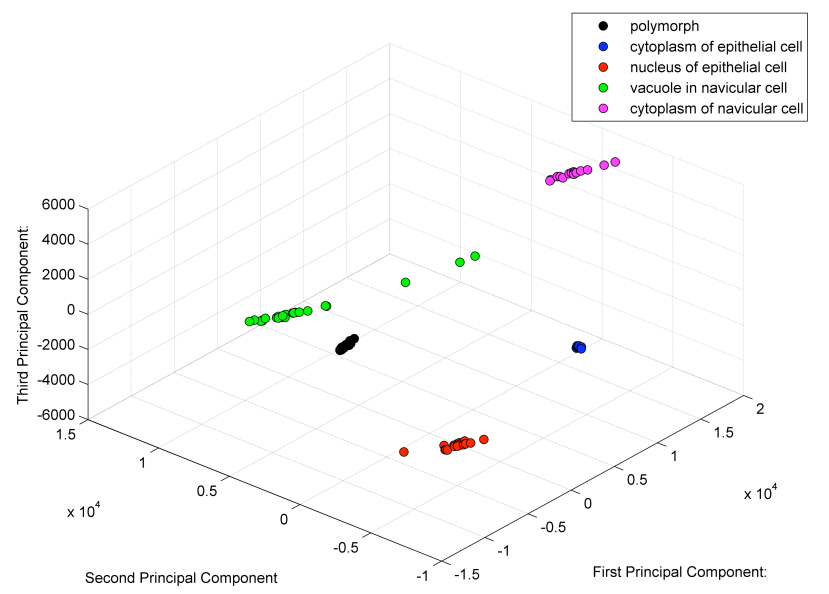

Fig. 3. PCA scatter plot demonstrates discrimination between cellular regions of cervical smear constituents.

\section{CONCLUSION}

This paper presents an overview of a range of activities in biophotonics and imaging currently in progress at the Focas Research Institute, involving applications of both FTIR and Raman spectroscopy and a range of chemometric and heuristic approaches to extract meaningful and useful measures from the hyperspectral data recorded during spectroscopic imaging. The application of such analytical techniques must be considered an integral and inseparable component of the application of vibrational spectroscopy in biomedical analysis.

\section{ACKNOWLEDGEMENTS}

The Focas Institute, DIT has been established under the Irish Higher Education Authority HEA Programme for Research in Third Level Institutions, Cycle 1 (1999-2001). The authors acknowledge funding through the Technology Sector Research (Strand 3) programme of the HEA, and HEA PRTLI Cycle 4 (National Biophotonics and Imaging Platform of Ireland (NBIPI)).

\section{REFERENCES}

[1] K. A. Janes and M. B. Yaffe, "Data-driven modelling of signal-transduction networks," Nat Rev Mol Cell Biol, vol. 7, pp. 820-8, Nov 2006.

[2] C. Matthaus, T. Chernenko, J. A. Newmark, C. M. Warner, and M. Diem, "Label-free detection of mitochondrial distribution in cells by nonresonant Raman microspectroscopy," Biophys $J$, vol. 93, pp. 66873, Jul 152007.

[3] C. Petibois and G. Deleris, "Chemical mapping of tumor progression by FT-IR imaging: towards molecular histopathology," Trends Biotechnol, vol. 24, pp. 455-62, Oct 2006.

[4] E. O. Faolain, M. B. Hunter, J. M. Byrne, P. Kelehan, H. A. Lambkin, H. J. Byrne, and F. M. Lyng, "Raman spectroscopic evaluation of efficacy of current paraffin wax section dewaxing agents," J Histochem Cytochem, vol. 53, pp. 121-9, Jan 2005.

[5] E. O Faolain, M. B. Hunter, J. M. Byrne, P. Kelehan, M. McNamara, H. J. Byrne, and F. M. Lyng, "A study examining the effects of tissue processing on human tissue sections using vibrational spectroscopy," Vibrational Spectroscopy, vol. 38, pp. 121-127, Jul 29 2005.

[6] F. M. Lyng, E. O. Faolain, J. Conroy, A. D. Meade, P. Knief, B. Duffy, M. B. Hunter, J. M. Byrne, P. Kelehan, and H. J. Byrne, "Vibrational spectroscopy for cervical cancer pathology, from biochemical analysis to diagnostic tool," Exp Mol Pathol, vol. 82, pp. 121-9, Apr 2007.

[7] X. Michalet, F. F. Pinaud, L. A. Bentolila, J. M. Tsay, S. Doose, J. J. Li, G. Sundaresan, A. M. Wu, S. S. Gambhir, and S. Weiss, "Quantum dots for live cells, in vivo imaging, and diagnostics," Science, vol. 307, pp. 538-44, Jan 282005.

[8] H. J. van Manen and C. Otto, "Hybrid confocal Raman fluorescence microscopy on single cells using semiconductor quantum dots," Nano Lett, vol. 7, pp. 1631-6, Jun 2007.

[9] R. Hardman, "A toxicologic review of quantum dots: toxicity depends on physicochemical and environmental factors," Environ Health Perspect, vol. 114, pp. 165-72, Feb 2006.

[10] P. Knief, C. Clarke, E. Herzog, M. Davoren, F. M. Lyng, A. D. Meade, and H. J. Byrne, "Raman spectroscopy - a potential platform for the rapid measurement of carbon nanotube-induced cytotoxicity," Analyst DOI: 10.1039/b821393c, 2009.

[11] A. D. Meade, F. M. Lyng, P. Knief, and H. J. Byrne, "Growth substrate induced functional changes elucidated by FTIR and Raman spectroscopy in in-vitro cultured human keratinocytes," Anal Bioanal Chem, vol. 387, pp. 1717-28, Mar 2007. 